\title{
Ayurvedic Biology - An Unbiased Approach to Understand Traditional Health-care System
}

\author{
S C LAKHOTIA* (D) \\ Editor-in-Chief, and Cytogenetics Laboratory, Department of Zoology, Banaras Hindu University, Varanasi \\ 221005 , India
}

Wisdom transmitted through generations in the form of experience based folk-lore and customs greatly influences the life styles practiced by the given society. Keeping fit and healthy is one of the basic instincts of all living systems and they are now known to employ a variety of proactive strategies to achieve it. Accordingly, human societies also developed customs and practices that were believed to maintain health and combat various illnesses. Thus every civilization across the world has evolved its traditional healthcare system which is handed down the generations through folk-lore and which continues to be enriched by newer experiences (Lakhotia, 2015). The traditional health-care systems in China (Traditional Chinese Medicine or TCM) and in India (Ayurveda) are the oldest (>4000 years) and most elaborate, thanks to extensive ancient literature being available about their philosophies and practices. These systems are widely practiced, not only in respective countries, but also across the globe.

In recent decades the experiment based "modern medicine" has made unbelievably rapid advances, largely because of the remarkably deeper understanding of life processes at cellular and subcellular levels. Equally fascinating developments in chemistry and other technologies have made it possible to replace the herbal drugs with precisely crafted molecules which in combination with equally novel diagnostic and drug-delivery methods have transformed the health-care systems during the past 4-5 decades. Notwithstanding the great strides that have succeeded in eliminating several deadly infectious diseases and in prolonging the average life span, there are serious issues due to novel life-style associated disorders which are not easy to cure by modern medicines; more worrisome are the sideeffects of many of the modern drugs and the increasingly high costs that such health-care systems demand.

Although the roots of modern medicine can be traced in the diverse traditional health-care systems (Valiathan, 2016), the modern and the traditional systems in the current scenario view each other with suspicion, often bordering on contempt. The feelings of contempt or suspicion on either side are not due to "conflict of interest", but seem to be rooted in the underlying approaches and philosophies. Modern medicine is experiment based and much of its medication is based on the rigours of molecular biology using a reductionist approach. On the other hand, traditional systems like the TCM and Ayurveda are largely based on experience of thousands of years with a holistic approach where any ailment in body is believed to be an outcome of some disturbance in the complex network that maintains homeostasis in the body. A major emphasis of the modern medicine is to cure a disease or disorder after its manifestation using a reductionist approach so that a given medicine/ treatment is specifically targeted to the immediate cause of disease/disorder. Ayurvedic philosophy on the other hand believes that its health-care practices are not directed to cure a disease but are primarily used to maintain a healthy state and thereby prevent disease. A healthy state is maintained by following some basic principles of life style, diet supplemented with some formulations/medications, and other exercises/practices that promote homeostasis between different body systems. Together, these are

*E-mail: lakhotia.eic.insa@gmail.com 
believed to prevent disease and permit healthy aging. Ayurvedic approach to health is claimed to be holistic since, analogous to contemporary "systems biology", it believes in the living body being a network of interacting components. Further, analogous to the cherished goal of the modern medicine to develop "personalized medicine", Ayurvedic as well as TCM systems claim that their approach is already personalized because they take into account a number of combinations of the individual's features and properties prior to defining the course of medication in relation to a given disorder/ailment. However, in spite of such comprehensive and attractive philosophies and practices that characterize systems like Ayurveda, a major limitation is that there is little experimental evidence about their mechanisms of actions which can be understood in the language of contemporary principles of science.

I recently participated in an Ayurvedic Congress where there was a session on Ayurvedic Biology with a view to bring the practitioners of Ayurveda and cell and molecular biologists together to facilitate greater mutual appreciation. While such concourses help thaw the ice, they also reveal why the desired results are not forthcoming easily. A major cause for the mutual suspicion and lack of appreciation between those practicing traditional and modern medicine/science, respectively, appears to lie primarily in the unwillingness to engage with each other and, thereby, appreciate and follow the "good" principles and practices of each while modifying or giving up the ones that do not qualify a rational experimental explanation. The major cause for the unwillingness to discuss lies in the different philosophies and vocabulary. Ayurvedic practitioners are not trained or exposed to the contemporary principles of biology, especially the enormous progresses in fields of genetics and genomics, cell and molecular biology, physiology etc. Unfortunately, many of them also appear to think that they do not need to re-interpret Ayurveda in light of the contemporary knowledge as they remain dogmatically faithful to the philosophies and principles of human body organization enunciated thousands of years ago by great scholars like Caraca, Sushruta and others. Practitioners of contemporary science and medical practices who are primarily trained in the reductionist approach, on the other hand, find it very difficult to correlate the principles and philosophies followed in traditional health-care systems with experimentally verifiable entities as known now. This generates justified or unjustified skepticism about many concepts and practices of the traditional medicine systems. Obviously, there is a strong need to correlate these different languages and philosophies to find the commonalities. Just as the practitioners of Ayurveda need to come out of their "shell" and begin to understand human biology as we understand today, the scientists and modern medicine practitioners need to read and appreciate the holistic approach of Ayurvedic health-care system and, thereby, reinterpret those principles in the current vocabulary. While an unbiased enquiry into the principles of Ayurveda as enunciated thousands of years ago is not to be taken as an insult to the wisdom of those ancient sages, those principles should also not be discarded just because they are old. We may never know the nature of research and experimental observations that the ancient scholars and sages may have carried out to define the principles and practices followed in Ayurveda and TCM but we can certainly rediscover them in terms of our increasingly deeper understanding of the molecular events spanning from sub-cellular to organismic and population levels. Then only we will be able to separate the distilled essence of experience-based traditional wisdom from myths that would have naturally grown around.

"A Science Initiative In Ayurveda" (ASIIA, see Valiathan in this issue, pp 13-19) has triggered some dialogue between geneticists, cell and molecular biologists, physicists, material scientists etc on one hand and the practitioners of Ayurveda on the other. As noted by Professor M S Valiathan in this issue, this initiative has already resulted in some exciting interactions and mutual appreciations. The ASIIA initiative has resulted in the formation of an "Ayurvedic Biology" group under the Science \& Engineering Research Board (Department of Science \& Technology, Govt. of India) to support basic science research in Ayurveda. An unbiased and deeper involvement of Ayurvedic practitioners in such experimental studies is essential to provide the much needed insights about the holistic health-care system which the scientists and modern medicine practitioners can examine and understand in the light of current experiment based approach. The recent Nobel Prize for the discovery of Artemisinin, based on the 
knowledge base of TCM, is a pointer to the success of synergistic studies involving different, and seemingly conflicting, approaches and philosophies. It is high time that the opportunities provided for such inter-disciplinary studies are extensively and intensively utilized to bring about a confluence of traditional and modern health-care systems (Lakhotia, 2015) so that the increasing menace of life-style and other chronic disorders can be faced successfully.

\section{References}

Lakhotia S C (2015) Exploring traditional medicine - attempt to validate layman's experience-based health-care systems across the world Proc Indian Natn Sci Acad $\mathbf{8 1}$ 1081-1085

Valiathan M S (2016) Ayurvedic Biology: the first decade Proc Indian Natn Sci Acad 82 13-19. 